JOURNAL OF COLLOID SCIENCE 16, 501-512 (1961)

\title{
ON THE INTERPRETATION OF ELECTROKINETIC POTENTIALS
}

\author{
J. Lyklema and J. Th. G. Overbeek \\ van 't Hoff Laboratory, State University of Utrecht, The Netherlands \\ Received January 27,1961
}

INTRODUCTION

Electrokinetic measurements are of considerable interest for the study of electrical double layers. It is customary to interpret electrokinetic data in terms of the so-called electrokinetic or $\xi$-potential. This is the potential of the slipping plane between the moving and the stationary phase, when the liquid far away from the interface is considered to be at zero potential.

The calculation of $\zeta$ from electrokinetic data is often uncertain because it involves the dielectric constant, $\epsilon$, and the viscosity, $\eta$, and these may have abnormal values in part of the double layer. If one tries to correlate $\zeta$ with $\psi_{0}$, the total potential drop across the double layer, more difficulties are encountered, such as uncertainties about the location of the slipping plane, the structure of the liquid inside the slipping plane, and discreteness of charges effects.

It is the purpose of this paper to contribute to the solution of these problems by considering the variability of the viscosity and dielectric constant in the double layer and proposing a model for the mechanics of the slipping process. We shall confine ourselves to aqueous solutions, taking electroosmosis and electrophoresis as the examples.

The Variability of the Dielectric Constant in the Electrical Double Layer

From double layer capacity evidence it is most likely that the dielectric constant in the inner region of the double layer is smaller than that of the bulk. This is due to saturation effects caused by the high field strengths in the double layer and to the structural influence of the adjacent phase. This decrease in $\epsilon$ can have a considerable influence on properties involving the inner region of the double layer (1-3).

It is therefore important in the establishment of the relation between $\psi_{0}$ and $\zeta$. Whether it also influences the relation between $\zeta$ and the electrophoretic mobility, $u$, depends on the actual value of $\epsilon$ in the outer region of the double layer. The following reasoning will show that in this case saturation effects may usually be neglected.

A formula for the dielectric saturation of water is given by Booth (4) 
based upon theories of Onsager and Kirkwood. This formula reads:

$$
\epsilon=n^{2}+\frac{28 N \pi\left(n^{2}+2\right) \mu}{3 \sqrt{73} E} \mathrm{~L}\left(\frac{\sqrt{73} E \mu\left(n^{2}+2\right)}{6 k T}\right),
$$

in which $N$ represents the number of water molecules per $\mathrm{cm}^{3}, n$ the refractive index, $\mu$ the dipole moment, $E$ the acting electric field strength, and $\mathrm{L}(z)$ the Langevin function. For low values of $z, \mathrm{~L}(z)$ may be replaced by the first term of its series expansion being $z / 3$. From Eq. [1] one sees that $\epsilon$ is independent of $E$ when this condition is fulfilled. Inserting the appropriate numerical values in Eq. [1] a quantitative relation between $\epsilon$ and $E$ can be found. The dielectric saturation is $2 \%$ for $E=5 \times 10^{5} \mathrm{v} . / \mathrm{cm}$. and $10 \%$ for $E=1.5 \times 10^{6} \mathrm{v} . / \mathrm{cm}$. Such high field strengths can be produced only by a combination of high potential and high ionic strength, and are rarely found outside the slipping plane. This is illustrated in Table I giving the $\zeta$-potentials required to reach the field strengths mentioned above.

From this table it may be concluded that it is mostly justified to use the bulk value of $\epsilon$ in the electrophoresis equation of Helmholtz-Smoluchowski:

$$
u=\frac{\epsilon F}{4 \pi \eta} \zeta
$$

where $F$ is the applied field strength.

\section{The Variability of the Viscosity in the Electrical DOUBLE LAYER}

A fully satisfactory theory for the dependence of the viscosity of liquids (especially water) on the strength of an applied electric field has not yet been given. Several workers investigated this dependency experimentally $(5-14)$. In all cases $\eta$ is found to increase with $E$. A quantitative formulation was given by Da.C. Andrade and Dodd (6):

$$
\frac{\eta_{E}-\eta_{E=0}}{\eta_{E=0}}=\frac{d \eta}{\eta}=f E^{2}
$$

TABLE I

Values of the Diffuse Double Layer Potential ( () above Which the Dielectric Saturation Exceeds $2 \%$ and 10\%, Respectively

(Gouy-Chapman picture; (1-1)-electrolytes)

\begin{tabular}{ccc}
\hline \multirow{2}{*}{ Electrolyte concentration } & \multicolumn{2}{c}{ Dielectric saturation of } \\
\cline { 2 - 3 } & $2 \%$ & $10 \%$ \\
\hline 1 molar & $15 \mathrm{mv}$. & $40 \mathrm{mv}$. \\
$10^{-2}$ molar & $94 \mathrm{mv}$. & $148 \mathrm{mv}$. \\
$10^{-4}$ molar & $210 \mathrm{mv}$. & $270 \mathrm{mv}$. \\
\hline
\end{tabular}


In this equation $\eta_{E=0}$ equals the viscosity with no field applied, and $f$ represents the so-called viscoelectric constant. The following values were found $(6)$ :

$$
\begin{aligned}
& \text { chloroform } f=1.89 \times 10^{-12} \mathrm{v}^{-2} \mathrm{~cm} .^{2} \\
& \text { amylacetate } f=2.74 \times 10^{-12} \mathrm{v}^{-2} \mathrm{~cm} .^{2} \\
& \text { chlorobenzene } f=2.12 \times 10^{-12} \mathrm{v} .^{-2} \mathrm{~cm} .^{2}
\end{aligned}
$$

Deviations of, for example, $10 \%$ in the viscosity for these liquids can therefore be expected to occur at field strengths of

$$
E>\sqrt{(10 f)^{-1}} \sim 2 \times 10^{5} \mathrm{v} . / \mathrm{cm} .
$$

This is much lower than the field strength required for a $10 \%$ change in $\epsilon\left(E>1.5 \times 10^{6}\right.$ volts $/ \mathrm{cm}$.). Such field strengths may occur outside the slipping plane. The provisional conclusion must be that Eq. [2] requires a correction for the variation of the viscosity, whereas variations in $\epsilon$ may be neglected.

The argumentation given above is restrieted to the three organic liquids mentioned. The viscoelectrie constant of water $\left(f_{w}\right)$ has not been measured. It can, however, be found from a theoretical reasoning.

To this purpose we start with Reynolds' equation for the viscosity of a liquid, written as

$$
\eta=A \exp \left(\frac{\alpha}{k T}\right)
$$

In this equation $A$ is a constant and $\alpha$ can be interpreted as the activation energy of viscous flow. When the dipolar molecules of the liquid are oriented in an electric field this activation energy increases with a quantity $\alpha_{\mathrm{el}}$, leading to:

where

$$
\eta+d \eta=A \exp \left(\frac{\alpha+\alpha_{e l}}{k T}\right)=\eta \exp \left(\frac{\alpha_{e l}}{k T}\right)
$$

$$
\alpha_{\mathrm{el} .}=B_{\mu^{2} E_{i}{ }^{2} .}
$$

$E_{i}$ is the internal field strength and $B$ is a structural specific constant of dimension [energy $]^{-1}$ and of the order $\left(k T^{T}\right)^{-1}$. It depends among other things on the coordination number and density of the liquid. For field intensities which are not too high the exponential in Eq. [5] may be expanded, retaining only two terms, which gives:

$$
\frac{d \eta}{\eta}=\frac{B \mu^{2}}{k T} E_{i}^{2}=\frac{B \mu^{2} G^{2}}{k T} E^{2},
$$

when $E_{i}=G E$. Comparison of [7] with [3] yields:

$$
f=\frac{B \mu^{2} G^{2}}{k T} \text {. }
$$

The factor $G$ is given by (15)

$$
G=\frac{3 k T+\mu R^{*}}{3 k T} \frac{1}{1-g_{\alpha}} \frac{3 \epsilon}{2 \epsilon+1},
$$


where $R^{*}$ is the reaction field;

$$
R^{*}=\frac{g}{1-g_{\alpha}} \mu,
$$

where $g$ is a reaction field factor and equal to $\frac{1}{a^{3}} \frac{2 n^{2}-2}{2 n^{2}+1}$ ( $a$ representing the radius of the spherical molecule); $\alpha$ is the polarizability and can be calculated with the corrected Lorenz-Lorentz equation (15)

$$
\frac{\left(n^{2}-1\right)\left(2 n^{2}+1\right)}{12 \pi n^{2}}=\frac{N_{\alpha}}{1-g \alpha} .
$$

By inserting the appropriate values in [9] $G$ can be calculated for water and the three organic liquids. With the aid of Eq. [8] the following values of $B$ are found:

$$
\begin{array}{ll}
\text { chloroform } & 0.186 \times 10^{15} \mathrm{erg}^{-1} \\
\text { amylacetate } & 0.154 \times 10^{15} \mathrm{erg}^{-1} \\
\text { chlorobenzene } & 0.114 \times 10^{15} \mathrm{erg}^{-1} \\
\text { Mean value } & 0.15 \times 10^{15} \mathrm{erg}^{-1}
\end{array}
$$

Though the structural properties of these liquids vary considerably their $B$ constants do not differ by more than a factor $5 / 3$. Therefore the use of the mean $B$-value will probably lead to a reasonable estimate for the viscoelectric constant for water:

$$
f_{w}=10.2 \times 10^{-12} \mathrm{v}^{-2} \mathrm{~cm} .^{2}
$$

This value of $f_{w}$ is used in the following section. As long, however, as $f_{w}$ is not measured independently its value remains somewhat uncertain (see also reference 13).

The influence of the double layer field on the viscosity is illustrated by Table II. Comparison with Table I shows that the influence of the field on the viscosity is much more pronounced than that on the dielectric constant. It is seen that viscosity corrections are necessary in many cases.

\section{Correction of the Helmholtz-Smoluchowski Equation FOR THE VARIABILITY OF THE VISCOSITY}

Consider a double layer at a flat interface, perpendicular to the $x$ axis in an electric field of strength $F$ parallel to this interface.

\section{TABLE II}

Values of the Diffuse Double Layer Potential ( $\zeta$ ) above Which a Viscosity Increase of $2 \%$ and $10 \%$, Respectively Is Found

(Gouy-Chapman picture; (1-1)-electrolytes; $f_{w}=10.2 \times 10^{-12} \mathrm{v}^{-2} \mathrm{~cm} .{ }^{2}$ )

\begin{tabular}{ccc}
\hline \multirow{2}{*}{ Electrolyte concentration } & \multicolumn{2}{c}{ Viscosity increase of } \\
\cline { 2 - 3 } & $2 \%$ & $10 \%$ \\
\hline $10^{-2}$ molar & $1.4 \mathrm{mv}$. & $3 \mathrm{mv}$. \\
$10^{-4}$ molar & $14 \mathrm{mv}$. & $29 \mathrm{mv}$. \\
\hline
\end{tabular}


Consider a volume element of thickness $d x$ and area of $1 \mathrm{~cm} .{ }^{2}$, also perpendicular to the $x$ axis. The field exerts a force $F \rho d x$ on this volume element if $\rho$ represents the space charge density. In the stationary state this force is just balanced by the force of viscous shear:

$$
F \rho d x+\frac{d}{d x}\left(\eta \frac{d v}{d x}\right) d x=0
$$

where $v$ is the rate of streaming.

Substituting Poisson's equation in the form

$$
\frac{d}{d x}\left(\epsilon \frac{d \psi}{d x}\right)=-4 \pi \rho
$$

we find

$$
-\frac{F}{4 \pi} \frac{d}{d x}\left(\epsilon \frac{d \psi}{d x}\right)+\frac{d}{d x}\left(\eta \frac{d v}{d x}\right)=0 .
$$

This equation can be integrated, utilizing the boundary conditions that far away from the surface $(x=\infty)$ the velocity gradient and potential gradient are both zero. One obtains:

$$
\frac{\epsilon F}{4 \pi \eta}\left(\frac{d \psi}{d x}\right)=\left(\frac{d v}{d x}\right)
$$

It is of interest to note that this equation is valid independent of the constancy of $\epsilon$ and $\eta$.

The second integration is now carried out with constant $\epsilon$ and leads to

$$
u=\frac{\epsilon F}{4 \pi} \int_{0}^{\zeta} \frac{d \psi}{\eta}
$$

where $u$ represents the velocity at the point $x=d$, where $\zeta=\psi_{d}$, and where there is no shear for $x<d$. Then $u$ is equal to the electrophoretic velocity.

The integration can be carried out if the relation between $\eta$ and $\psi$ is given. To this purpose we assume that $\eta$ is correctly given by Eq. [3] written in the form

$$
\eta_{x}=\eta_{0}\left(1+f\left(\frac{d \psi}{d x}\right)^{2}\right)
$$

where $\eta_{0}$ is the bulk viscosity and $(d \psi / d x)$, the double layer field strength, is given by the Gouy-Chapman theory as

$$
\frac{d \psi}{d x}=\sqrt{\frac{32 \pi c R T}{1000 \epsilon}} \sinh \left(\frac{z \mathrm{~F} \psi}{2 R T}\right)
$$


where $c$ is the concentration of the $(z-z)$-valent electrolyte in moles/liter and $\mathbf{F}$ is the Faraday.

From [16], [17], and [18] it follows that

$$
u=\frac{\epsilon F}{4 \pi \eta_{0}} \int_{0}^{\zeta} \frac{d \psi}{1+\frac{32 \pi c R T}{1000 \epsilon} f \sinh ^{2}\left(\frac{z \mathrm{~F} \psi}{2 R T}\right)},
$$

which for sake of simplicity can be written as

$$
u=\frac{\epsilon F}{4 \pi \eta_{0}} \int_{0}^{\zeta} \frac{d \psi}{1+A \sinh ^{2}\left(\frac{\beta \psi}{2}\right)} .
$$

The integration can be carried out analytically. It leads to three different equations, depending on the value of $A$.

The solution for $A<1$ reads:

$$
\begin{aligned}
& u= \frac{\epsilon F}{4 \pi \eta_{0}} \frac{1}{\beta \sqrt{1-A}} \\
& \cdot \ln \frac{\left(2 \exp \left(\frac{z \mathrm{~F} \zeta}{R T}\right)+\left(\frac{4}{A}-2\right)-\frac{4}{A} \sqrt{1-A}\right)}{\left(2 \exp \left(\frac{z \mathrm{~F} \zeta}{R T}\right)+\left(\frac{4}{A}-2\right)+\frac{4}{A} \sqrt{1-A}\right)} \frac{(1+\sqrt{1-A})}{(1-\sqrt{1-A})} .
\end{aligned}
$$

The solution for $A>1$ reads:

$$
\begin{aligned}
u=\frac{\epsilon F}{4 \pi \eta_{0}} & \frac{2}{\beta \sqrt{A-1}} \\
& \cdot\left\{\arctan \frac{2 \exp \left(\frac{z \mathrm{~F} \zeta}{R T}\right)+\left(\frac{4}{A}-2\right)}{\left.\frac{4}{A} \sqrt{A-1}-\arctan \frac{1}{\sqrt{A-1}}\right\} .} .\right.
\end{aligned}
$$

For $A=1$ both solutions approach to

$$
u=\frac{\epsilon F}{4 \pi \eta_{0}} \frac{2}{\beta} \operatorname{tgh}\left(\frac{z \mathbf{F} \zeta}{2 R T}\right) .
$$

For small values of $\zeta$ or the concentration $(A \infty c)$ the following approximated solution of Eq. [20] may be useful:

$$
u \approx \frac{\epsilon F}{4 \pi \eta_{0}} \cdot \int_{0}^{\zeta}\left\{1-A \sinh ^{2}\left(\frac{\beta \psi}{2}\right)\right\} d \psi=\frac{\epsilon F}{4 \pi \eta_{0}}\left[\zeta-\frac{A}{2 \beta}\{\sinh (\beta \zeta)-\beta \zeta\}\right],
$$

which ean be further approximated for small $\zeta$ to

$$
u \approx \frac{\epsilon \zeta F}{4 \pi \eta_{0}}\left[1-\frac{A \beta^{2}}{12} \zeta^{2}\right] .
$$


These equations show clearly the relation with the original equation of Helmholtz and Smoluchowski.

An illustration of these solutions is presented in Fig. 1. On the ordinate axis the difference is plotted between the potential of the slipping plane, $\zeta$ (real) and the "observed" potential, $\zeta_{\text {obs. }}$ (calculated simply from Eq. [2]).

The figure holds for monovalent electrolytes at $25^{\circ} \mathrm{C}$. and $f_{w}=10.2 \times$ $10^{-12} \mathrm{v} \cdot{ }^{-2} \mathrm{~cm} .{ }^{2}$. The approximation [24] is indistinguishable from the drawn curves within the $5 \%$ correction limit. The curve for $c=0.035 \mathrm{~mole} / \mathrm{l}$. corresponds with $A=1$ (Eq. [23]); the $10^{-1}$ curve corresponds with $A>1$ (Eq. [22]); and the other curves correspond with $A<1$ (Eq. [21]). For low salt content the correction is expected to stay always below $5 \%$. For somewhat higher salt contents ( $>10^{-2}$ mole/l.) the correction may reach $20 \%$ and even exceed this value in the case of the unfavorable combination of high $\zeta$ and high concentration. The correction limits the electrophoretic mobility regardless of the potential in the double layer. (See below, in particular, Fig. 4.)

\section{Some Considerations concerning the Slipping Process}

One of the most important aims of electrokinetic measurements is the correlation between $\psi_{0}$ and the electrophoretic velocity, $u$. If the electrical double layer consists of a molecular condenser with a potential drop $\psi_{0}-\psi_{\delta}$ and a Gouy-Chapman diffuse double layer with a potential drop $\psi_{\delta}$ and if we assume that no slipping occurs within the molecular condenser, the problem resolves itself into the establishment of the relation between $\psi_{0}$ and $\psi_{\delta}$ and the relation between $u$ and $\psi_{\delta}$. The first relation is not relevant

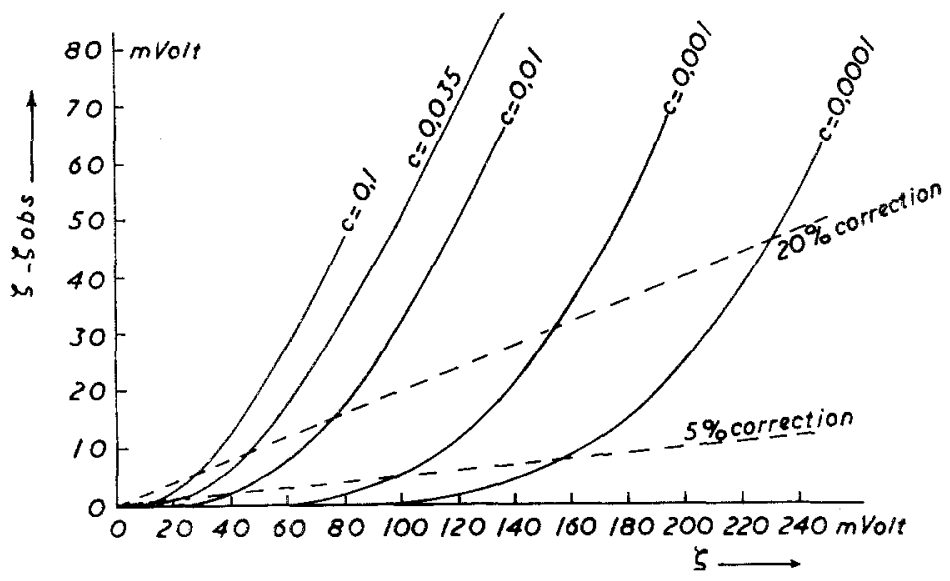

Frg. 1. Correction of the Helmholtz-Smoluchowski equation for the increase of the viscosity in the electrical double layer at different concentrations ( $c$ in moles/ liter) of (1-1) electrolytes. $f_{w}=10.2 \times 10^{-12} \mathrm{v.}^{-2} \mathrm{~cm}^{2} ; 25^{\circ} \mathrm{C}$. The dotted lines indicate $5 \%$ and $20 \%$ correction. 
for this paper; the calculation can, for example, be accomplished assuming that no specific adsorption occurs (16). We are here concerned with the second relation only.

From the preceding sections it is felt that the classical concept of a discrete slipping plane is too simple. There exists a slipping layer with a gradual transition from bulk fluidity to zero fluidity. This variation in viscosity may be due to the high electrostatic field strength, but this is not necessarily the only effect. A direct influence of the interface on the viscosity is also conceivable, in particular, an increase of the viscosity to very high values.

Discontinuities in the fluidity can be imagined in systems where solvated molecules are oriented in a peculiar way, behaving as an individual phase, for example, in so-called autophobic systems (17).

The actual relation between the observed electrokinetic potential, $\zeta_{\text {obs. }}$, and $\psi_{\delta}$ depends on the relative importance of the surface influence and double layer field influence on viscosity. As a matter of illustration we will discuss two extreme cases, one in which the viscosity is affected only by the viscoelectric effect and the other in which there is an immobilized layer of considerable thickness close to the surface.

\section{a. No Direct Influence of the Surface on the Viscosity}

For extremely low potentials the slipping plane is identical with the surface $\left(\zeta=\psi_{\delta}\right)$. With increasing potential the viscoelectric effect causes the

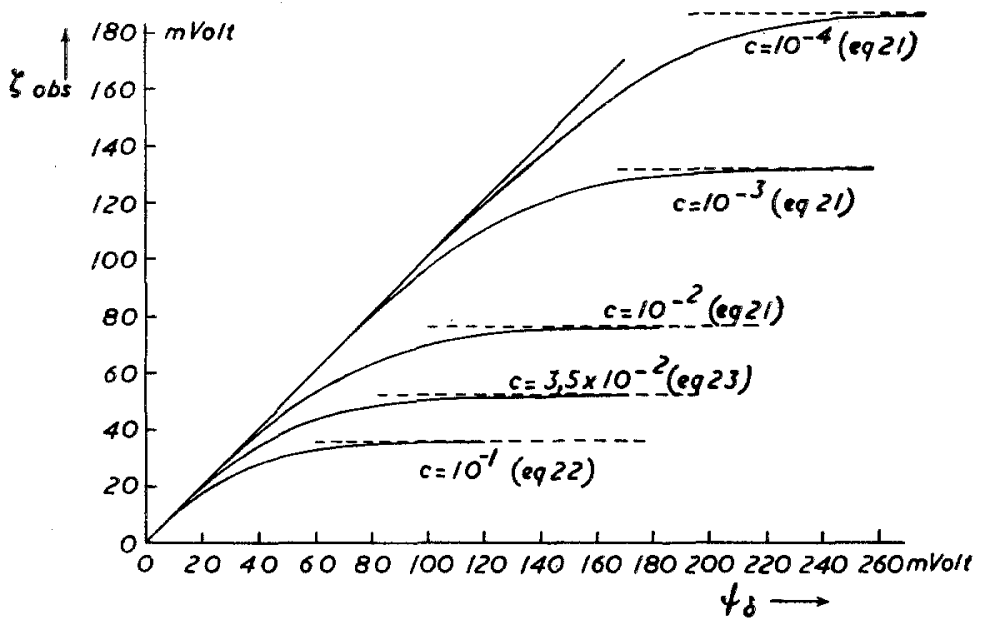

FIG. 2. Observed electrokinetic potential as a function of the surface potential in the absence of structural influence of the surface on the viscosity. Dotted lines: limiting values. 
discrete slipping plane to be gradually replaced by a slipping layer of finite thickness. At last the viscosity can be so high that virtually no slip takes place at the interface. The slipping layer, the location of which is determined by the field strength, moves in outward direction with increasing potential, and this will eventually lead to a constant electrophoretic mobility (independent of $\psi_{\delta}$, but dependent on the concentration). This dependency is illustrated in Fig. 2. The limiting values have been calculated from Eqs. [21]-[23] for $\zeta\left(=\psi_{\delta}\right) \rightarrow \infty$. In Fig. 3 these limiting values are plotted as a function of the concentration. For low concentrations the relationship is linear with a slope of $58 \mathrm{mv}$. per tenfold increase in the concentration, but for higher concentrations the slope decreases.

1). At high $\zeta$-potential the influence of the relaxation effect on electrophoresis should not be neglected. It also lowers the mobility.

2). In the given treatment $f_{w}$ was considered to be independent of the concentration. This is probably an oversimplification (18). The influence of the concentration on $f_{w}$ will change the curve of Fig. 3 , and detailed analysis of the dependence of $\zeta_{\text {obs. }}$ from $\log c$ must be considered with a good deal of reservation.

Figure 2 can be exemplified by observed $\zeta$-potentials on negative AgI which appear to be more or less independent of pI, i.e., of the surface potential $\psi_{0}$ over an appreciable range far from the zero point of charge. This followed from electrophoretic data (19-21), from streaming potentials (22), and also from sedimentation potentials at high $\psi_{0}$ as measured by Rutgers and Nagels (23), at least if correction was made for the relaxation effect. This constancy may be explained by the present theory. Recent data on

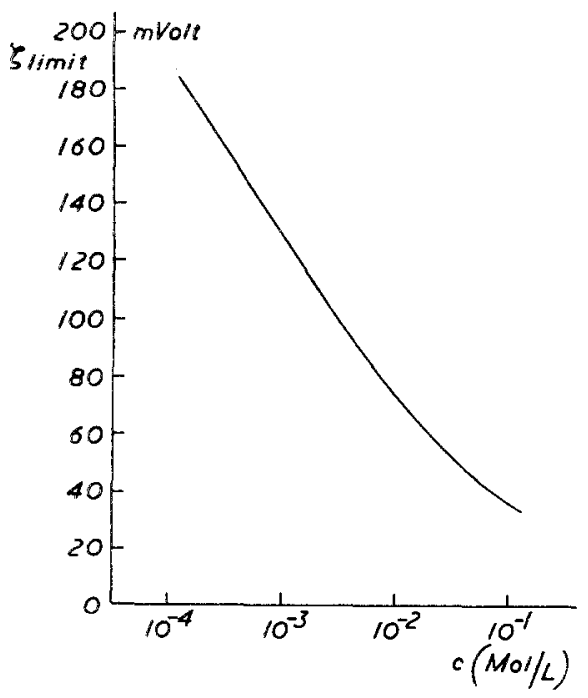

FIG. 3. Limiting values of $\zeta_{\text {obs. }}$. 
the $\zeta$-potential at the oil-water interface as determined by Haydon (24) are also in qualitative agreement with the present picture especially with Fig. 2. For low potentials $\zeta$ and $\psi_{0}$ were identical. For higher $\psi_{0} \zeta$ obs. remained more or less constant. For high $\psi_{0}$ it decreased to some extent. This decrease must be explained by other factors, such as specific adsorption of ions and dependence of $f_{w}$ on the composition of the solution. The constant zeta potentials are dependent not only on the concentration but also on the nature of the electrolyte and the nature of the soap (24); this-at least in part-may be due to the influence of concentration and nature of the solute on $f_{w}$.

\section{b. Strong Influence of the Surface on the Viscosity}

We assume now that several layers of molecules of the liquid are immobile with respect to the surface. The field strength outside this layer is already so low that the viscosity does not deviate very much from $\eta_{0}$. This case can be treated as the classical, discrete slipping plane at a fixed distance (d) from the outer Helmholtz plane, with small viscosity correction $\zeta_{\text {obs. }} \sim$ $\zeta_{(\mathrm{real})}=\psi_{d}$.

When specific adsorption of ions in the adhered layer may be neglected, $\zeta=\psi_{d}$ and $\psi_{\delta}$ are related through the equation

$$
\exp \left(\frac{z \mathrm{~F} \zeta}{2 R T}\right)=\frac{\exp (\kappa d)+\tanh \left(\frac{z \mathrm{~F} \psi_{\delta}}{4 R T}\right)}{\exp (\kappa d)-\tanh \left(\frac{z \mathrm{~F} \psi_{\delta}}{4 R T}\right)}
$$

where $\kappa$ is the reciprocal Debye length.

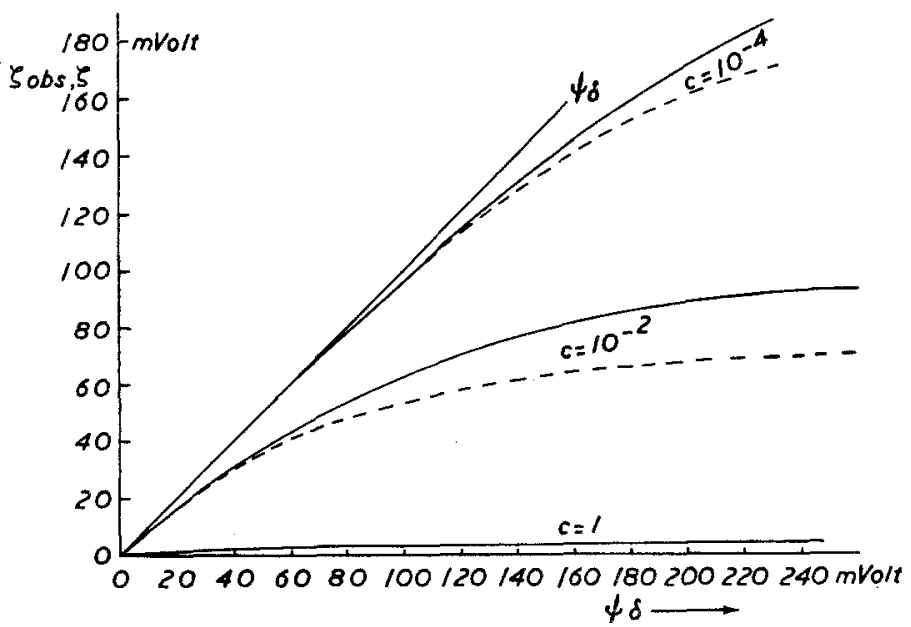

FIG. 4. Relation between the observed zeta potential, $\zeta_{\text {ous. }}(----)$, the potential of the slipping plane $\zeta(-)$, and the potential of the outer Helmholtz plane, $\psi_{\delta}$. 
The relation between $\zeta$ and $\psi_{\delta}$ is shown in Fig. 4 for three concentrations, $z=1$ and $d=10 \mathrm{~A}$. The dotted curves represent the $\zeta_{\text {obs. }}$ values, corrected for the viscoelectric effect with the help of Fig. 1.

For very high $\psi_{\delta}$ the potential of the slipping plane approaches a constant value (independent of $\psi_{\delta}$ ) given by

$$
\exp \left(\frac{z \mathbf{F} \zeta}{2 R T}\right)=\frac{1+e^{-\kappa d}}{1-e^{-\kappa d}} .
$$

This constant value of $\zeta$ is seldom reached in practical cases. However, the electrophoretic mobility can become independent of $\psi_{\delta}$ at much lower potentials as a consequence of the viscoelectric correction. Comparison of Fig. 4 with Fig. 2 shows that in the case of Fig. 4 the constant electrophoretic mobilities are found at much higher surface potentials. Indeed the rate with which the electrophoretic velocity grows constant is a measure for the thickness of the solvated layer (influence of the surface) and for the magnitude of the viscoelectric constant (influence of the double layer viscosity). The limiting values of $\zeta_{\text {obs }}$, are, however, equal for both cases.

The relation between $\zeta_{\text {abs. }}$ and $c$ is governed by the influence of the concentration on the relations [21]-[23] and [26]. This influence is given in Fig. 5 for three values of $\psi_{\delta}$. It shows that the relation between $\xi$ and $\log c$ is not linear. The slope varies with concentration and potential and is generally smaller than $58 \mathrm{mv}$. per tenfold change in concentration.

The observed zeta potential decreases more strongly with $\log c$ than the real one. At extremely high $\psi_{\delta}$ (limiting case) the slope is equal to that of Fig. 3. It should be noted that $\psi_{\delta}$ itself is usually a decreasing function of $c$; this makes the slope of the curve relating $\zeta_{\text {obs }}$ and $\log c$ for constant $\psi_{0}$ steeper than that for constant $\psi_{\delta}$.

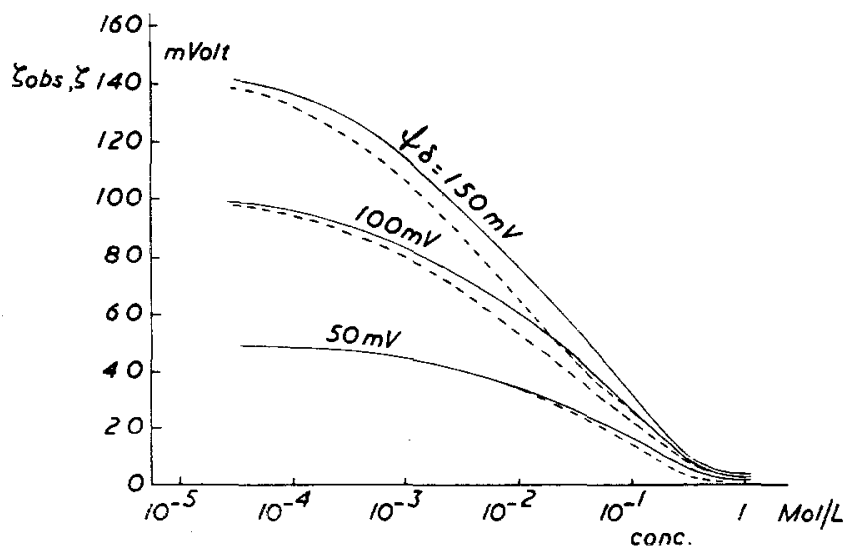

FIG. 5. Solid curves: relation between $\zeta$ and $\log c$ for constant slipping plane at 10 A (1-1 electrolytes; diffuse double layer, for three values of $\psi_{\delta}$ ). Dotted curves: corrected $\zeta$-potential ( $\left.\zeta_{\text {obs. }}\right)$. 
An example of curves of this type are the $\zeta \log c$ curves for glass, as measured electroosmotically by Rutgers and De Smet (25).

The main point we wish to stress is that the effects calculated in this paper are sufficiently large and agreement with general experience is good enough to make a determination of the viscoelastic constant for water and aqueous solutions highly desirable.

\section{Summary}

The variation of the dielectric constant and the viscosity in the electrical double layer are considered. The Helmholtz-Smoluchowski equation for the electrophoretic mobility requires only insignificant correction for variations in the dielectric constant; the variation in the viscosity can lead to considerable corrections, which increase with surface potential and concentration. Equations are given for the relation between electrophoretic mobility and surface potential and are illustrated by two examples. The concept of the slipping plane should be replaced by a slipping layer of finite thickness. At high surface potentials, the electrophoretic mobility is independent of the potential but depends on the electrolyte concentration.

\section{REFERENCES}

1. Conway, B. E., Bockris, J. O'M., and Ammar, I. A., Trans. Faraday Soc. 47, 756 (1951).

2. Brodowsky, H., and Strehlow, H., Z. Elektrochem. 63, 262 (1959).

3. Sparnaax, M. J., Rec. trav. chim. 77, 872 (1958).

4. Bоoтh, F., J. Chem. Phys. 19, 391, 1327, 1615 (1951).

5. Andrade, E. N. Da. C., And Dodn, C., Proc. Roy. Soc. (London) A187, 296 (1946).

6. Andrade, E. N. Da. C., And Dodd, C., Proc. Roy. Soc. (London) A204, 449 (1951).

7. Alcock, E. D., Physics 7, 126 (1936).

8. Buörnståhl, Y., and Snellman, K. O., Kolloid-Z. 78, 258 (1937).

9. Calker, J. v., And Aubke, B., Z. Physik 131, 443 (1952).

10. Chernyuk, A. K., Akad. Nauk S.S.S.R., Otdel. Tekh. Nauk Inst. Mashinovedeniya, Soveshchanie Vyazkosti Zhidkost. i Kolloid. Rastvorov 2, 62 (1944); Chem. Abstr. 40, 3315 ${ }^{1-9}(1946)$.

11. Снмuтin, M. S., Uchenye Zapiski Stalingrad Gosudarst. Pedagog. Inst. 1953 (No. 3) 92; Chem. Abstr. 50, 4572i (1956).

12. Hart, J., Physica 23, 795 (1957).

13. Hart, J., J. Chem. Phys. 29, 960 (1958).

14. Osamu Kamura, I., J. Chem. Soc. Japan 64, 895, 937 (1943).

15. Bötrcher, C. J. F., "Theory of Electric Polarisation," especially 11 sections. Elsevier, Amsterdam, Houston, London, New York, 1952.

16. Grahame, D. C., Chem. Revs. 41, 441 (1947).

17. Hare, E. F., and Zisman, W. A., J. Phys. Chem. 59, 335 (1955).

18. Haydon, D. A., Private communication.

19. Troelstra, S. A., And Kruyt, H. R., Kolloid-Z. 101, 182 (1942).

20. Ottewill, R. H., and Rastogi, M. C., Private communication.

21. Bi.sterbosch, B. H., Unpublished results.

22. Pravdić, V., And Mirnik, M., Croat. Chem. Acta 32, 75 (1960).

23. Rutgers, A. J., and Nagels, P., J. Colloid Sci. 13, 140 (1958).

24. Haydon, D. A., Proc. Roy. Soc. (London) A258, 319 (1960).

25. Rutgers, A. J., ANd Smet, M. DE, Trans. Faraday Soc. 41, 758 (1945). 